\title{
Virtual Heritage Expo: Joint presentation of research work and historic artefacts
}

\author{
Johanna Leissner \\ Fraunhofer-Gesellschaft for the \\ advancement of Applied Research \\ Brussels Office, Rue Royale 94 \\ B-1000 Bruxelles, Belgium \\ johanna.leissner@zv.fraunhofer.de
}

\author{
Martin Papirowski \\ men@work Media Services S.R.L. \\ Sperantei 28 \\ 020994 Bucharest \\ Romania \\ papirowski@menatworkmedia.com
}

\author{
Ralf Schäfer, Oliver Schreer \& \\ Christian Weißig \\ Fraunhofer Heinrich Hertz Institute \\ Einsteinufer 37 \\ 10587 Berlin, Germany \\ ralf.schaefer/oliver.schreer/christian.weissig
}

@hhi.fraunhofer.de

\begin{abstract}
Fraunhofer-Gesellschaft for the advancement of applied research has created a "Virtual Heritage Expo" as a contribution to the European Year of Cultural Heritage 2018: It consists of several virtual exhibition halls presenting the research and technology development performed at Fraunhofer-Gesellschaft for the protection of historic monuments and artefacts. Over hundred individual objects, 3D models of significant artefacts from 20,000 years of human history, virtual projection screens and complex installations are used in this exhibition. The Virtual Heritage Expo can be visited in two ways: By individual visits using Virtual Reality glasses or as a multi-user experience, by watching a virtual tour on an ultra-high resolution (2000 x 7000 pixel) curved screen. Different technologies have been used to create the content for these virtual experiences: Render engines for high resolution CGI, Lidar and 3D scanners for scanning 3D objects, $360^{\circ}$ cameras with up to $10.000 \times 3.500$ pixel resolution and a volumetric studio, to create dynamic 3D models of persons.
\end{abstract}

Virtual museum. World heritage. Presentation of research work. 3D models of artefacts.

\section{INTRODUCTION}

Already a quarter of a century ago, Manfred Koob, architect and university teacher at TU Darmstadt recognised one of the greatest dangers to the World Heritage: People! Cultural mass tourism represents a great threat to the most important monuments of humanity from a conservation perspective. But how can we strategically address this decay of the world cultural heritage and protect it from people without excluding them? An unsolvable task - at a first glance. The FraunhoferGesellschaft, Europe's largest organisation for applied research with over 70 institutes and 27,000 scientists is proposing an innovative solution: We call it the Virtual Heritage Expo. It is the world's first virtual exhibition of applied research dedicated to the world heritage. We have created a complex of exhibition halls in which we present our research and development work together with historic monuments and artefacts as a virtual exhibition. We use multimedia, over hundred individual objects, 3D models of significant artefacts from
20,000 years of human history, virtual projection screens and complex installations. This expo combines virtual 3D worlds in a breath-taking architecture with stereoscopic $360^{\circ}$ expeditions to important monuments such as the Green Vault in Dresden, the ancient city of ruins Pompeii, the Cologne Cathedral or Göbekli Tepe, the first temple of humankind.

The Virtual Heritage Expo can be visited in two ways: By using Virtual Reality glasses, for which a special exhibition booth for four visitors has been constructed and as a multi user experience, an ultra-high resolution (2000 x 7000 pixel) projection on a curved $180^{\circ}$ screen, which transports the viewer into the virtual world without wearing glasses.

\section{ARTISTIC CONCEPT}

The construction and design of virtual spaces for Virtual Reality experiences is still a pioneering technology. There is no empiricism that has grown 
over decades; most approaches of VR creators are still quite experimental. Although there are a number of examples of virtual museums such as "The Kremer Museum" (Kremer), there is no installation known to the authors, where both new technologies and cultural heritage are on exhibition together in a virtual environment, therefore a new concept was required. The primary task of Virtual Heritage Expo is to communicate didactically technologies and scientific developments in the service of the preservation of the world cultural heritage on one hand and to raise awareness for the topic and its problems on the other hand.

\subsection{The VR experience}

The goal of the VR experience is to illustrate comprehensibly that cultural heritage protection needs innovative research and technology development while also entertaining the recipient, offering him a strong immersive experience, unforgettable minutes in a virtual world.

These two requirements have to be considered and implemented in the concept, which however creates a problem: Many museum curators subordinate the space to the exhibits. The architecture of the museum space, the lighting design and the public guidance are exclusively oriented towards the exhibits, the artefacts. An extreme example is the concept of the "Dark Room", in which the showcases look like islands of light in a nocturnal sea. This philosophy is in contrast to the idea of the Wilhelmine or Victorian museum palaces, enormous domed buildings in which the exhibits degenerate into miniaturised decorative objects.

VR has the potential to combine these two philosophies. However, one has to consider that Virtual Reality is not a copy of reality, but it creates its own reality. The virtual space is by no means constrained by the architecture of the real world. It has no static, economic or scientific limitation in size, form or design, which is one of the reasons, why VR is so overwhelming for the user.

Similarly there is no need for fully realistic representations, explanatory pieces, exhibits and artefacts have to be comprehensible, and they must beguile the recipient. Therefore, we combined video shots and $360^{\circ}$ video with virtual spaces, 3D models of scanned objects and persons in order to put reality and virtual reality on a par.

\subsection{The architecture of the exhibition}

The exhibition halls are grouped around a central hall building, the Big Hall. The architecture of the interiors of the Virtual Heritage Expo is not a copy of an existing architectural concept, but rather a reminiscent of computer-generated film backdrops of science fiction productions. Measured in terms of the size of the human being, the exhibition halls correspond to the size of Gothic cathedrals. The BIG HALL, with a floor area of around 20,000 square metres, corresponds to the floor area of the Basilica of Saint Peter, after all one of the largest and most important churches in the world.

An exception is the so-called Roggersdorf Hall, which represents a fully simulated church in Upper Bavaria. The church building is surrounded by a real landscape with sea cliffs, low mountain ranges, lakes and forests. A biotope with an area of perhaps eight square kilometres in the real world, where this biotope occupies only part of the hall area (see Figure 1).

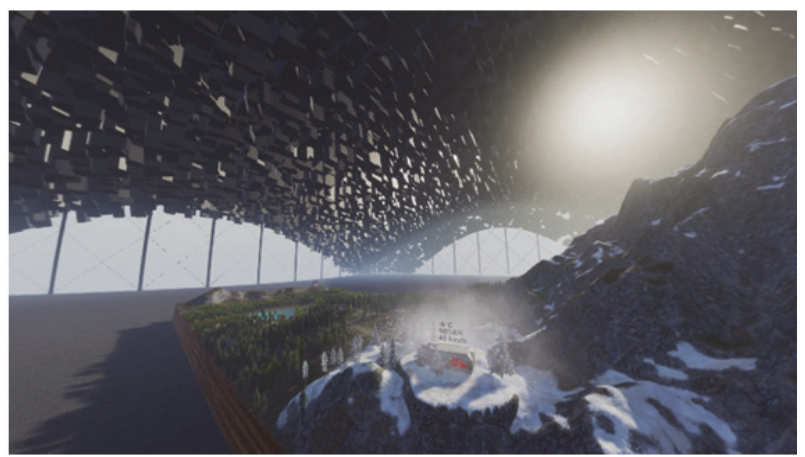

Figure 1: Landscape of Roggersdorf.

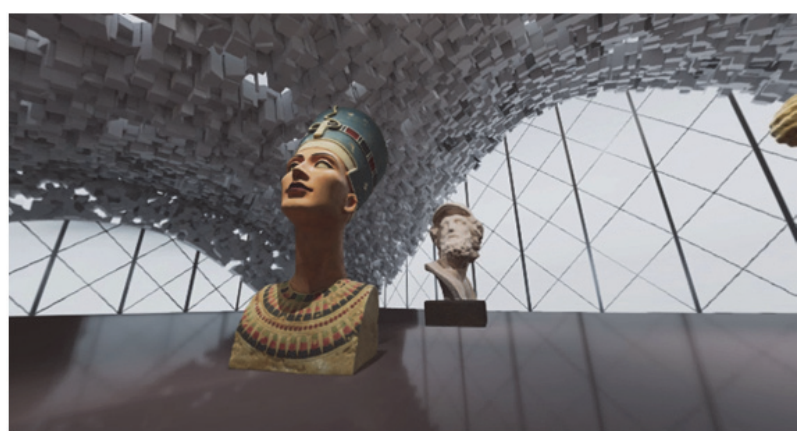

Figure 2: Egyptian pharaoh busts.

Much bigger than in reality are also the historical artefacts that dominate the exhibition concept. Examples are a Chinese terracotta soldier, an Egyptian pharaoh busts (see Figure 2) or the Venus of Willendorf, which dates back to more than 20,000 years and represents one of the oldest art objects of mankind. In the BIG HALL more than 40 exhibits with dimensions of over 40 meters height can be seen.

In the CultLab 3D hall, which represents the automated 3D scanning technology, the recipient himself becomes a scan object The recipient is following the procedural steps of the world's only fully automatic scanning line from the subjective perspective of an artefact (see Figure 3). 


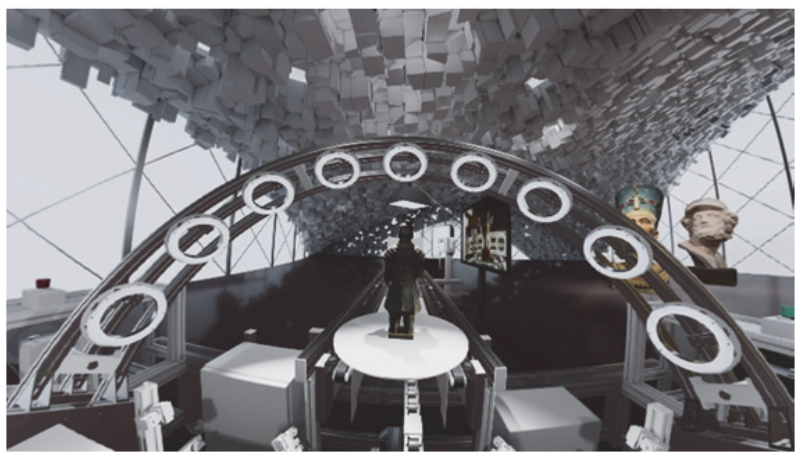

Figure 3: CultLab3D hall

\section{CONTENT CREATION}

The advantage of Virtual Reality is that multiple sources of 2D and 3D information can be combined in a highly creative way. Therefore, various content production technologies have been used ranging from classical 3D modelling and 2D footage integration towards much more advanced techniques such as ultra-high-resolution panoramic video capture and volumetric video.

\subsection{Scanning of 3D objects}

The high quality 3D reconstruction of cultural heritage artefacts has been performed with the CultLab 3D Scanner by Fraunhofer IGD. CultLab3D is an extendable, multi-modular scanning facility using the next generation of autonomous and compliant robots as well as optical scanning technologies. The system consists of two scanning units (CultArc3D, CultArm3D) connected by a tray conveyor system. The entire acquisition process for geometry and texture of an object takes less than ten minutes on average, at a resolution in the submillimetre range. CultLab3D is currently designed for high-precision 3D acquisition of objects with up to $50 \mathrm{~kg}$ in weight and up to $60 \mathrm{~cm}$ in length, width and height. Some other high quality $3 \mathrm{D}$ models have been received from other Fraunhofer institutes, which used laser scanning methods

\subsection{D reconstruction of sites}

Some elements in the Virtual Heritage Expo are modelled in 3D in high detail and texture using standard 3D modelling tools. For example, the Roggersdorf Hall, which surrounds a real landscape with sea cliffs, low mountain ranges, lakes and forests, was modelled manually (see Figure 1). The user can fly across this miniaturised landscape and experience Roggersdorf at different weather conditions.

\subsection{0 degree video}

360 -degree video is an adequate technology to allow the viewer to experience historic sites virtually. It can be used for both presentations forms, VR-glasses and immersive panoramic cinemas. Compared to photogrammetric reconstructions, 360-degree video allows the reproduction of dynamic scenes. It is obvious that this possibility can provide a higher authenticity, however it causes a higher technical effort for content generation.

For applications demanding high quality, Fraunhofer $\mathrm{HHI}$ developed a whole family of $2 \mathrm{D}$ and 3D omnidirectional cameras called OmniCam360. The OmniCam360 is a mirror-based multi-camera system that allows the recording in a $360^{\circ}$ panoramic format. The sophisticated segment-wise combination of mirrors and cameras enables a parallax-free setup, which allows perfectly stitched images even for live applications. The OmniCam-version used for the productions in the context of the Virtual Heritage Expo is using a combination of $11 \mathrm{HD}$ respective UHD-cameras and provides a resolution of 10.000 by 3.500 pixels at a frame rate of up to $60 \mathrm{~Hz}$. The field of view covers 360 by 120 degrees.

Fraunhofer $\mathrm{HHI}$ has developed a Real Time Stitching Engine (RTSE) for the processing of the individual image segments to generate a seamless overall panoramic image. The RTSE supports all the processing steps needed, including warping, stitching, blending and colour matching. Since the RTSE provides outputs of the stitched panorama in different formats and resolutions, it can also be used for the live preview during the film production.

The final composition of the content has been coordinated by Fraunhofer Brussels and by Fraunhofer $\mathrm{HHI}$, which also provides the viewing facilities.

The OmniCam-360 has already been used for various panorama productions over the last years. In the context of the Virtual Heritage Expo, the first series of productions has been done in the Green Vault in Dresden (Figure 4), the ancient city of Pompeii (Figure 5) and the historical optical Fraunhofer glass workshop at Benediktbeuern.

\subsection{Volumetric video}

Volumetric video is another element used in the Virtual Heritage Expo in order to provide a realistic and convincing representation of humans in the virtual world. Volumetric video is regarded worldwide as the next important development step in the field of media production. 


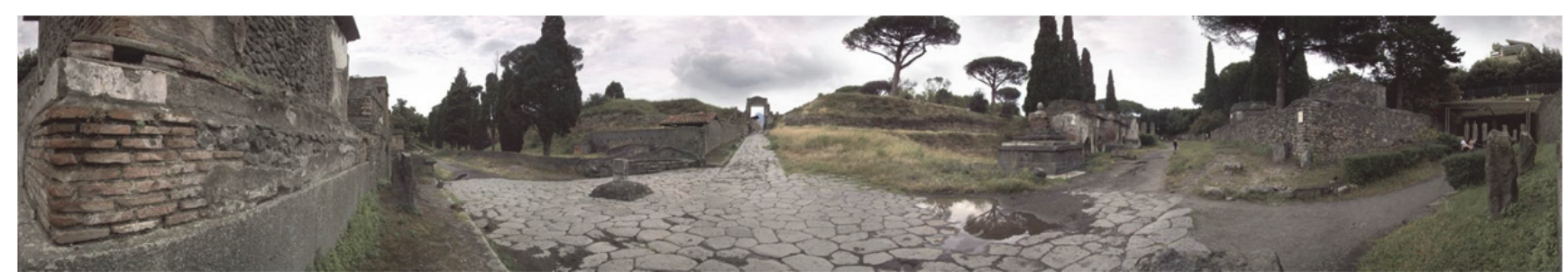

Figure 4: Funerary monuments of the Necropolis of "Porta Nocera" in Pompeii.

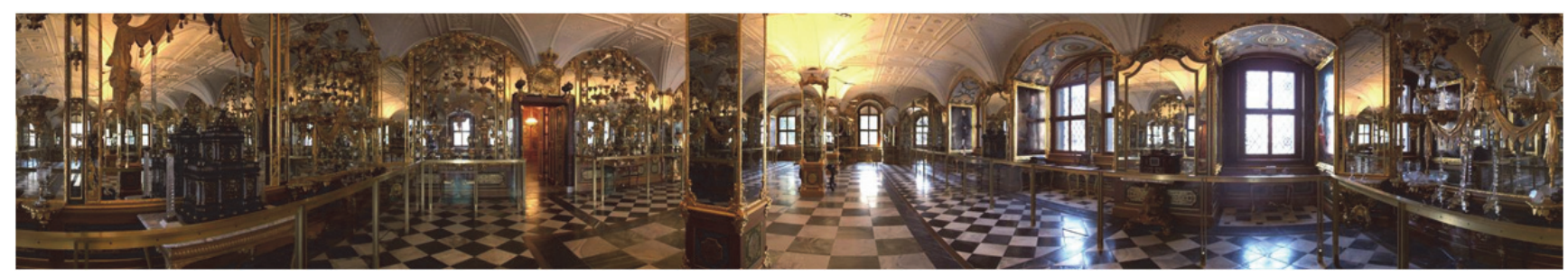

Figure 5: Green Vault in Dresden.

The main concept is to capture real persons with multiple cameras simultaneously and create naturally moving dynamic 3D models, which can be observed from arbitrary viewpoints in the virtual scene. Fraunhofer $\mathrm{HHI}$ has developed a novel capture system, which is based on 32 highresolution cameras arranged as stereo pairs capturing a moving person in 360 degree (see Figure 6). A complex 3D video processing software for volumetric video, the so-called 3D Human Body Reconstruction (3DHBR), finally produces a sequence of meshes as dynamic 3D models that can then be integrated into common render engines such as Unity or Unreal. In Figure 7, left, a sample mesh is shown, which is then integrated in a virtual reality scene as shown in Figure 7 , right. In contrast to classical animation of virtual characters, facial expressions as well as moving clothes are captured from video information and reconstructed at high geometrical detail and texture quality (Schreer et al.).

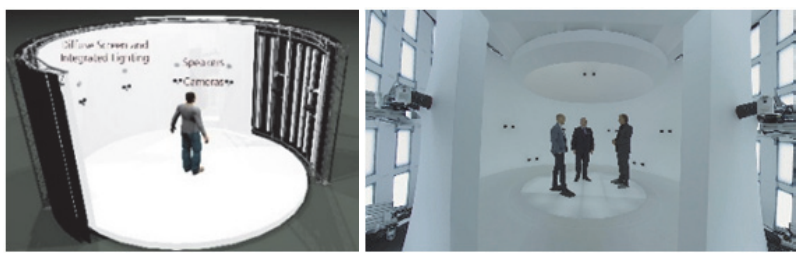

Figure 6: Drawing of the capture and light stage (left) and first prototype (right).
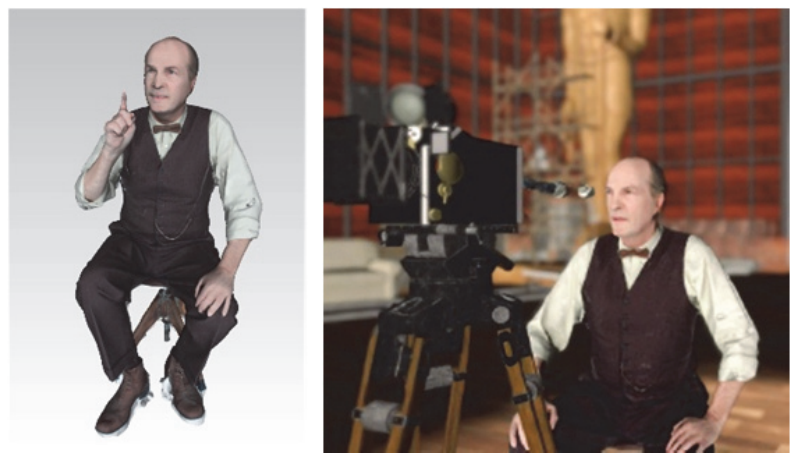

Figure 7: Resulting 3D model (left) and integrated model in virtual reality scene (right).

\section{COMPOSITING OF THE EXPERIENCE}

Originally, the Virtual Heritage Expo was designed as an interactive VR experience to allow the user to walk around in the virtual space and explore the different halls. The experience was developed in Unreal and targeted for Oculus Rift as one of the standard head mounted displays for VR. However, the complexity of the complete Virtual Heritage Expo becomes too high for an interactive experience due to the large number of $3 D$ elements. Therefore, a guided 360-degree stereoscopic 3D video based VR experience has been derived from the available 3D models. As Unreal cannot provide the necessary rendering quality for 360 degree video, the big halls have been imported to Houdini and high quality ray tracing is used to achieve best possible video quality. 
However, the interactive VR experience is still under consideration, but the overall concept needs to be further developed in order to allow a fluent interaction with minimised re-loading of 3D data.

\section{THE VIEWING EXPERIENCE}

\subsection{VR glasses}

For the VR experience, the 3D models are rendered with Houdini in a resolution of $3840 \mathrm{x}$ 1920 for the left and right eye. The same applies for the panoramic video content. The resulting MPEG-video can be experienced in any capable player for stereoscopic 360-degree video. For demonstration purposes, a movable cube has been designed that allows parallel viewing experience for four users (see Figure 8). At each side, an Oculus Rift is available to watch the VR experience. Four state-of the art gaming PCs support fluent display and high quality graphics rendering. The whole cube can be easily transported and therefore shown in different locations and events of the Fraunhofer-Gesellschaft.

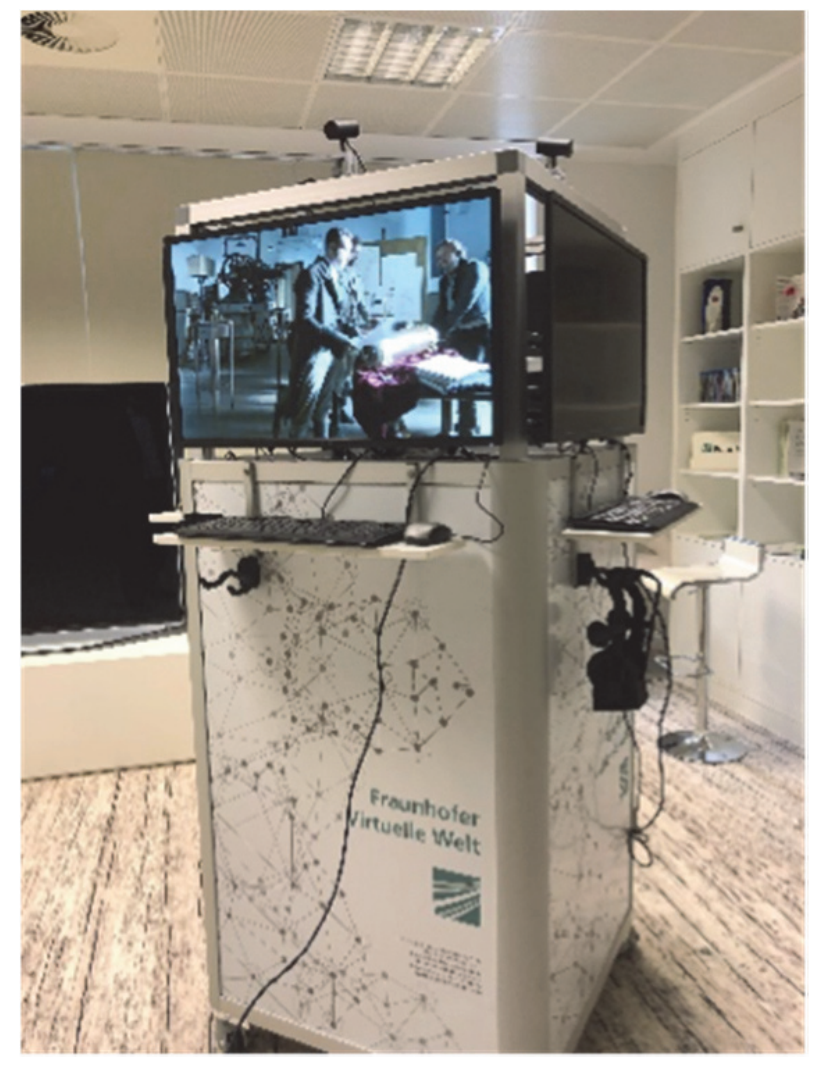

Figure 8: VR presentation cube.

\subsection{TiME Lab}

A further option to present virtual objects, historic monuments and artefacts in a realistic manner the usage of immersive rooms, where the audience is surrounded by the scene. Such panorama presentations have a long tradition; panorama paintings in huge rotundas have already been used in the 18th and 19th century for spectacular presentations of scenes of war, religion or landscape.

An authentic experience, in which the audience feels immersed into the virtual scenes, requires reproduction technologies for both, acoustic and visual presentation, that support quality features similar to those which are used to in real life.

With these requirements, Fraunhofer $\mathrm{HHI}$ developed an immersive presentation platform called "Tomorrow's immersive Media Experience (TiME) Lab". It is a panoramic cinema with a footprint of $8 \mathrm{~m}$ by $8 \mathrm{~m}$, equipped with a 180 -degree curved screen with a width of $12 \mathrm{~m}$ and a height of $3,4 \mathrm{~m}$. A multi-projection system using $14 \mathrm{HD}$ projectors provides $2 \mathrm{D}$ and $3 \mathrm{D}$ images with a resolution of $7,000 \times 2,000$ pixels (see Figure 9).

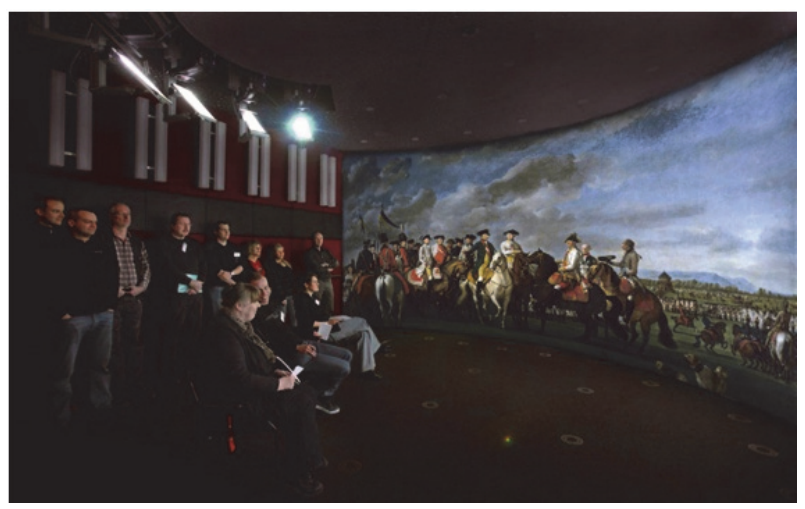

Figure 9: TiME Lab with presentation of a painting.

The TiME-Lab is also equipped with a spatial sound system in order to provide an authentic immersive audio presentation. It works as a wave field synthesis system (WFS) using 140 loudspeakers. WFS is an object-based sound system, which enables audio reproductions with a precise mapping of the sound objects to their visual sources to avoid perception conflicts. In addition, the acoustic characteristics of rooms or environments such as churches or concert halls can be realistically reproduced.

In comparison to VR glasses, immersive rooms as the TiME-Lab provide more natural multi-user experiences, because people can see each other while communicating. It is obvious that such kind of natural communication during the experience provides an additional benefit especially for education and training purposes.

\section{SUMMARY}

The Fraunhofer-Gesellschaft has developed a virtual exhibition, in which applied research 
dedicated to the world heritage is presented together with important artefacts of world heritage. This exhibition combines video shots, $360^{\circ}$ video, virtual spaces and 3D models of scanned object and persons. Virtual Heritage Expo is based on the artistic concept developed by Martin Papirowski, but technologies from different Fraunhofer institutes have been used to create this virtual exhibition. It can be experienced either on VR glasses or in an immersive viewing room, called the TiME Lab.

The final composition of the content has been coordinated by Fraunhofer Brussels and by Fraunhofer $\mathrm{HHI}$, which also provides the viewing facilities.

\section{REFERENCES}

CultLab3D (2019) http://www.cultlab3d.de (retrieved 10 April 2019).

Kremer (2019)

http://www.thekremercollection.com/the-kremermuseum/ (retrieved 10 April 2019).

Schreer O., Feldmann I., Ebner T., Renault S., Weissig Ch., Tatzelt D. and Kauff P. (2018) Advanced Volumetric Capture and Processing, Proc. of International Broadcasting Convention, Amsterdam, The Netherlands, September 2018. 